\title{
Mediación narrativa: técnicas y método para resolver conflictos en las organizaciones
}

Harvey Peña-Sandoval*

\footnotetext{
Magíster en Ciencias para el Análisis y la Resolución de Conflictos. Catedrático de la Maestría en Derecho Contractual, Facultad de Derecho, Universidad Santo Tomás, Bogotá, Colombia. Correo electrónico: harbeyps@gmail.com
}

Recibido: 24 de mayo del 2015

Aprobado: 17 de julio del 2015

Cómo citar este artículo: Harvey PeñaSandoval. Mediación narrativa: técnicas y método para resolver conflictos en las organizaciones. DIXI 22. Diciembre 2015.

Pág. 25. doi: http://dx.doi.org/10.16925/ di.v17i22.1234

\section{Resumen}

Propósito: este artículo da a conocer las técnicas y el método de la mediación narrativa como un mecanismo de resolución de conflictos en un contexto organizacional. Descripción: a fin de cumplir este objetivo, primero se define la mediación narrativa, después se presentan los supuestos de la teoría narrativa aplicada al conflicto, se describen las técnicas para la intervención en la mediación narrativa, se detallan las etapas de una mediación y finalmente se exponen algunas conclusiones sobre esta. Punto de vista: se basa en la mediación como un método de la disciplina de la resolución de conflictos, la cual a su vez se fundamenta en las teorías narrativas del conflicto y su resolución. Conclusión: la mediación narrativa permitirá a los empleados y funcionarios de empresas y entidades solucionar sus conflictos, de tal manera que las partes puedan crear historias de respeto, colaboración y reconocimiento mutuo. Se requiere un compromiso entre la academia y las organizaciones para desarrollar la mediación narrativa en Colombia.

Palabras clave: conflicto organizacional, mediación narrativa, resolución de conflictos, teoría narrativa. 


\title{
Narrative Mediation: Techniques and Method for Resolving Conflicts in Organizations
}

\begin{abstract}
Purpose: This article addresses the techniques and methods of narrative mediation as a conflict resolution mechanism in an organizational context. Description: To fulfill this objective, narrative mediation is first defined, after which the suppositions underlying the narrative theory as applied to conflict are presented. Techniques for intervention in the narrative mediation are then described, the stages of a mediation are specified and finally conclusions are stated. Point of view: Based on mediation as a method of the discipline of conflict resolution, which is in turn based on narrative theories of conflict and its resolution. Conclusion: Narrative mediation enables employees and officials of companies and entities to resolve their conflicts, so that the parties can create narratives of respect, collaboration and mutual recognition. There must be commitment by academia and organizations to develop narrative mediation in Colombia.
\end{abstract}

Keywords: organizational conflict, narrative mediation, conflict resolution, narrative theory.

\section{Mediação narrativa: técnicas e método para resolver conflitos nas organizações}

\section{Resumo}

Propósito: este artigo descreve as técnicas e o método da mediação narrativa como um mecanismo de resolução de conflitos num contexto organizacional. Descrição: a fim de atingir esse objetivo, primeiro define-se a mediação narrativa, depois se apresentam as hipóteses da teoria narrativa aplicada ao conflito, posteriormente descrevem-se as técnicas para a intervenção na mediação narrativa, detalham-se as etapas de uma mediação e finalmente expõem-se algumas conclusões sobre esta. Ponto de vista: baseia-se na mediação como um método da disciplina da resolução de conflitos, a qual, por sua vez, fundamenta-se nas teorias narrativas do conflito e de sua resolução. Conclusão: a mediação narrativa permitirá aos empregados e funcionários de empresas e entidades solucionar seus conflitos, de tal maneira que as partes possam criar histórias de respeito, colaboração e reconhecimento mútuo. Isso requer um compromisso entre a academia e as organizações para desenvolver a mediação narrativa na Colômbia.

Palavras-chave: conflito organizacional, mediação narrativa, resolução de conflitos, teoria narrativa. 


\section{INTRODUCCIÓN}

La literatura en español sobre la mediación como un mecanismo alternativo de solución de conflictos (MASC) es amplia y se ha basado especialmente en la mediación con enfoque facilitativo y transformativo. Sin embargo, poco se ha escrito sobre la mediación narrativa en nuestro idioma. Este artículo busca promover la mediación en Latinoamérica, así como presentar la mediación narrativa en un contexto organizacional como un recurso con el que cuentan los empleados y empleadores de entidades públicas y empresas para resolver sus conflictos con la ayuda de un mediador.

En el contexto de la mediación existen varias corrientes. Tres de las más conocidas son la mediación facilitativa, ${ }^{1}$ la mediación transformativa, ${ }^{2}$ y la mediación narrativa. ${ }^{3}$ En la mediación facilitativa — adaptada del método de negociación de Harvardel objetivo es producir resultados prudentes en forma eficiente y amistosa (este método es denominado negociación según principios o negociación con base en méritos). ${ }^{4}$ El objetivo de la mediación transformativa es el mejoramiento de las propias partes, en comparación con lo que eran antes (en este tipo de mediación se alcanza el éxito cuando las partes como personas cambian para mejorar a través del crecimiento en las dos dimensiones del desarrollo moral: la capacidad de fortalecer el yo y la capacidad de relacionarse con otros). ${ }^{5}$ Por último, en la mediación narrativa se busca ayudar a las personas en conflicto a separarse de la historia conflictiva en la que se encuentran y construir una nueva de respeto, colaboración y reconocimiento mutuo. ${ }^{6}$

En Colombia, el tipo de mediación más conocido ha sido el facilitativo, dada la gran difusión que han hecho los capacitadores de mediadores en la formación de estos. Por el contrario, la mediación narrativa es poco conocida en el país y, por tanto, se desconocen sus potencialidades en la resolución de los conflictos organizacionales. La mediación narrativa tiene una amplia aplicabilidad en la cultura organizacional colombiana, especialmente por la importancia que se le da a la oralidad en la construcción de las percepciones e imaginarios que se crean en las entidades públicas y empresas del país.

La teoría narrativa afirma que los conflictos son historias, el hombre es lo que dice y esto refleja su forma de entender la vida a través de las palabras que utiliza. ${ }^{7}$ Las personas viven sus conflictos a través de narrativas gracias a las cuales las partes se perciben como contrarias. En este punto es importante tener presente el concepto de conflicto desde una perspectiva narrativa: el conflicto es entendido desde afuera como un todo inevitable producto de la diversidad, más que la expresión de intereses o necesidades personales. ${ }^{8} \mathrm{La}$ definición del conflicto será entonces el marco conceptual que le permitirá al mediador intervenir en los conflictos de las partes. De esta manera, si el conflicto es la diferencia entre las narrativas, la gestión del conflicto debe ser a través de las narrativas mismas.

Para efectos del desarrollo de la mediación narrativa en el contexto institucional, a continuación se presentan los supuestos de la teoría narrativa que se aplican al conflicto y la mediación. Acto seguido, se describen las seis técnicas de intervención en los conflictos en una mediación narrativa. Posteriormente se detallan las etapas que se deben seguir a fin de desarrollar una mediación de tipo narrativo como un método de resolución de conflictos $y$, finalmente, se exponen algunas reflexiones a manera de conclusión.

\section{SUPUESTOS DE LA TEORÍA NARRATIVA APLICADA AL CONFLICTO}

\footnotetext{
MEDIACIÓN: cóMo AFRONTAR EL CONFLICTO MEDIANTE LA REVALORIZACIÓN Y EL RECONOCIMIENTO. Granica. (1996).

3. Sara Cobb. Narrative mediation working group. (Clase atendida por el autor). Abril 15 de 2011. Arlington, Vancouver, EE.uU.; Véase John Winslade. NARRATIVE MEDIATION: A NEW APPROACH TO CONFLICT RESOLUTION. Jossey-Bass. (2000).

4. Cf. Roger Fisher, William Ury y Bruce Patton, supra, nota 1. Pág. 11.

5. Cf. Robert A. Baruch y Joseph P. Folger, supra, nota 2. Págs. 134-135.

6. Concepto de mediación narrativa basado en John Winslade. Welcome letter. NARRATive AND CONFlict: Explorations in Theory and Practice. Diciembre de 2013. Págs. 1-3.

1 Véase Roger Fisher, William Ury y Bruce Patton. iSí, DE ACuERdo!: Cómo NEGOCIAR SIN CEDER. Norma. (1993).

2. Véase Robert A. Baruch y Joseph P. Folger. LA PROMESA DE LA
}

Antes de detallar las técnicas y el método de aplicación de la mediación narrativa, es necesario comprender los supuestos de este tipo de mediación. Lo anterior en

\footnotetext{
7. Véase John Winslade y Gerald Monk. Practicing NARraTIVE MEDiation: LOOSENING THE GRIP OF CONFLiCT. JosseyBass. (2008); y John Winslade. Welcome letter. Narrative AND Conflict: Explorations in Theory and Practice 1. Diciembre de 2013. Págs. 1-3.

8. Winslade, supra, nota 3. Pág. 41
} 
conformidad con el modo en que ha sido estructurada esta mediación, basada en teorías narrativas ${ }^{9}$ y cuyo fundamento teórico permite su práctica.

\section{Narrativa del conflicto y narrativa alternativa}

Es habitual entre las ciencias del conocimiento establecer un lenguaje común, en el cual las definiciones y los conceptos juegan un rol importante para la comprensión y aplicación de áreas propias de cada disciplina. La mediación narrativa no es la excepción y para esto existen dos conceptos fundamentales: narrativa del conflicto y narrativa alternativa. La primera hace referencia a la historia que las personas cuentan sobre su conflicto, la forma como narran lo que les sucede cuando están en una situación conflictiva y la manera como definen y entienden su conflicto. El conflicto ha atrapado a las partes y como efecto puede producir en ellas confusión, dolor, violencia, etc. Así como el conflicto toma a las partes y se dan consecuencias en principio no deseadas, estas también lo rechazan y buscan algo diferente; es en este momento cuando entra el concepto de narrativa alternativa. La narrativa alternativa es lo contrario a la narrativa del conflicto, es la historia en la que las partes se encuentran de nuevo en una relación de respeto, colaboración y legitimación mutua. Es una historia diferente a la del conflicto, a través de la cual los involucrados deciden cambiar lo que les ha sucedido y se comprometen a trabajar conjuntamente en la construcción de un camino que los lleve a un horizonte en el que se perciban en armonía. ${ }^{10}$

El conflicto puede ser tan poderoso que logra que las partes vivan en función de él. Esto se materializa, por ejemplo, cuando al preguntar a alguien “¿cómo estás?", la respuesta tiende a ser la narrativa

9. Mohammed D. Cherkaoui. Civilians, pundits, and the mediatized ideology. CIVILIANS AND MODERN WAR: ARMED CONFLICT AND THE IDEOlogy of violence. Routledge. (2012); Winslade, supra, nota 3; Sara Cobb. Speaking of Violence: THE POLITICS AND POETICS OF NARRATIVE DYNAMICS IN CONFLICT RESOLUTION. Oxford University Press. (2013); María Pía Lara. NARRATING EVIL: A POSTMETAPHYSICAL THEORY OF REFLECTIVE JUDGMENT. Columbia University Press. (2007); Hilde Lindemann. Damaged identities, NARRATIVE RePair. Cornell University Press. (2001); Gregory Alonso Pirio. Journalism and HIV. Lessons from the Frontline of Behavior Change Communication in Mozambique. Eds. Rafael Obregón y Silvio Waisbord. The HANDBook of GLOBaL HeAlth COMmunication. Wiley-Blackwell. (2012). Págs. 426-443; Solon Simmons. The ECLIPSE OF EQUALITY: ARgUing AMERICA ON MEET THE PRESS. Stanford University Press. (2013).

10. Véase Solon Simmons. The eclipse of equality: Arguing America on meEt the PRess. Stanford University Press. (2013). Págs. 72-89. del conflicto. Las personas llevan consigo mismas el conflicto a sus casas y otros espacios; en otras palabras, las personas se levantan y acuestan con el conflicto. Como ejemplo del impacto del conflicto en las personas se pueden considerar los temas sobre los cuales los colegas de trabajo conversan en sus almuerzos $\left({ }_{i}\right.$ Es el conflicto en la oficina la historia sobre la cual versa el diálogo del almuerzo?). Por otra parte, es posible evidenciar el cambio hacia una narrativa alternativa en el momento en que las personas que eran parte de un conflicto mediado hablan con otros colegas de la organización, y en este proceso describen a su otrora contraparte como alguien legítimo y con el cual tienen una relación respetuosa y colaborativa.

\section{Metanarrativas}

El conflicto puede ser consecuencia del choque entre la identidad y la cultura de una persona o un grupo con otros, contradicción que se puede producir entre las metanarrativas de las partes. Una metanarrativa es una regla social que las personas interiorizan sobre la forma en la cual debería organizarse el mundo, cómo deben ser las personas y la manera en la cual funcionan las cosas. Las metanarrativas son creaciones culturales y se acompañan de valores que definen a los otros. Por ejemplo, en una organización se puede pensar que "el buen trabajador es aquel que se queda después del horario de trabajo". Esta idea puede ser muy arraigada en la cultura organizacional, y se percibe cuando una persona sale a la hora en la que termina su horario de trabajo y sus colegas lo recriminan con comentarios tales como "cómo sale temprano, yo nunca lo logro"; “¿ya se va?”; o "parece funcionario público". Esta metanarrativa también se manifiesta en las conversaciones entre compañeros de la oficina, por ejemplo cuando un empleado constantemente manifiesta que tiene mucho trabajo y le toca salir muy tarde en la noche, y cómo esto representa su compromiso con la empresa. En fin, las metanarrativas son formas por medio de las cuales las personas construyen su identidad, las relaciones y sus roles en una comunidad. ${ }^{11}$

\section{Características de las narrativas}

Los conflictos como historias que contamos comparten las características de las narrativas, entre las cuales se encuentran:

- Trama. Los conflictos tienen una línea de tiempo en la que hay un comienzo (relación inicial de las

11. Id. Pág. 58. 
partes), un cambio (causas del conflicto), una complicación (momento en que surge el conflicto), y un desenlace (el cual puede ser un acuerdo resultado de una mediación).

- Temas. Los conflictos como historias tienen temas que las partes identifican como tipos de conflictos; por ejemplo, en una oficina pueden existir conflictos relacionados con acoso laboral, discriminación, abuso e inequidad, los cuales sumados llenan de contenido la narrativa del conflicto.

- Contexto. Los conflictos se desarrollan en espacios en los que las partes protagonizan la historia conflictiva. El contexto puede ser la familia, el trabajo, la comunidad, el país, etc. Dependiendo del contexto las partes desarrollan sus roles: padre, madre, hijo o hija en la familia; empleado, empleador o compañero en la oficina; vecino, líder comunitario o administrador en la comunidad; inmigrante, autoridad o nativo en el país.

- Personajes. Las partes en un conflicto cumplen roles cuyas complejas relaciones están inmersas en la narrativa del conflicto, y usualmente los papeles que desempeñan son antagónicos. Unas partes se identifican en el conflicto como víctimas y verán a sus contrarios como victimarios; otros pueden sentirse abusados y sus contrapartes serían los abusadores, etc. ${ }^{12}$

\section{Multiplicidad de narrativas}

La narrativa del conflicto es una historia compleja dada la multiplicidad de temas, contextos y personajes. No existe un conflicto "simple" o "fácil", toda vez que la vida de las personas y la forma en que estas se identifican y relacionan hacen del conflicto el punto de encuentro de muchos factores. Un conflicto sobre el ascenso de un empleado en una organización no es solamente un tema de ganar y perder. Debajo de la parte externa del conflicto puede subyacer una historia de discriminación e inequidad, resultado de una larga trama de relaciones en las que una persona percibe el no haberle otorgado la promoción como una consecuencia de una venganza por algo que hizo varios años atrás. De otro lado, las historias que una parte cuenta de la otra, en principio, se entienden como totalizantes de manera que la contraparte es algo parecido a un demonio o ser desprovisto de características humanas. Sin embargo, es imposible que alguien pueda ser definido como totalmente malo o villano.

12. Véase Cobb, supra, nota 3.
Seguramente en la narrativa existen también elementos legitimadores que posicionan al otro como bueno. Asimismo, la multiplicidad de las narrativas conlleva a que algunas historias sean dominantes y otras sean subordinadas (aquellas cuyas voces han sido acalladas). ${ }^{13}$

\section{Las personas no son el problema, el problema es el problema}

Uno de los aspectos complejos en el manejo de un conflicto es asociarlo con la persona con la que se tiene el conflicto. En otras palabras, la persona y el conflicto se convierten en una sola entidad. Si bien es cierto que las personas son las que ejecutan las acciones, desde la teoría narrativa de los conflictos el interés está enfocado en sus efectos nocivos y la percepción de las partes sobre este. El conflicto se debe situar en medio de las partes a fin de que estas de manera conjunta cooperen, decidan abandonarlo y construyan una historia alternativa. En este mismo orden de ideas, el conflicto debe ser externalizado para que las personas involucradas puedan legitimarse mutuamente. La idea se resume en la frase coloquial: "las personas no son el problema, el problema es el problema". Esto es, se debe concentrar la atención en el problema independientemente del sujeto parte del conflicto. Así, para superar el conflicto, el enfoque debe estar en lo que este les ha hecho a las personas (como si este fuera un tercero), y no en lo que las personas se han hecho entre sí. ${ }^{14}$

\section{TÉCNICAS EN LA MEDIACIÓN}

\section{NARRATIVA}

Al diseñarse la mediación narrativa como método de resolución de conflictos, las técnicas empleadas para llevar a cabo esta estrategia hacen parte de las herramientas que los mediadores aplican al dialogar con las partes y facilitar la mediación. En cuanto a las técnicas, puede ser útil entenderlas como una caja de herramientas. Cada herramienta tiene un propósito, no existe un manual en el que se establezca el orden y el paso a paso para aplicar cada técnica (herramienta), y esta por sí sola no logra el cometido de la obra (el cual para este caso es lograr que las partes

13. Cf. John Winslade y Michael Williams. SAFE AND PEACEFUL SCHOOLS: ADDRESSING CONFLICT AND ELIMINATING VIOLENCE. Corwin. (2012).

14. Winslade y Monk, supra, nota 7. Pág. 12. 
construyan una historia alternativa). Las técnicas no llevan a las partes a la solución de un conflicto: ayudan al mediador a conducir la mediación a fin de obtener unos resultados parciales en las partes y su narrativa. Las técnicas están asociadas al tipo de mediación y por esto las técnicas que se presentan a continuación tienen un enfoque narrativo.

\section{Doble escucha}

Esta técnica permite al mediador identificar elementos de la narrativa alternativa que subyace en la narrativa del conflicto. Cuando las personas cuentan lo que les está pasando con el conflicto (narrativa del conflicto), usualmente ellas lo dicen en términos negativos hacia la contraparte. Esta información puede ser anotada por el mediador con el fin de consignar literalmente lo que las partes afirman. Si se toma esa información y se transcribe en términos positivos, es posible extraer lo que las personas tal vez desean que pase pero no expresan (narrativa alternativa).

Por ejemplo, en el caso de un conflicto entre dos compañeros de trabajo, en el cual uno de ellos afirma: "él es un mal trabajador que llega tarde, se va temprano y se la pasa perdiendo el tiempo hablando por teléfono" (narrativa del conflicto literal). En esta frase se podría interpretar la narrativa alternativa de la siguiente manera: "quisiera que él fuera un buen trabajador que llega a tiempo, se va a tiempo y aprovecha el tiempo laboral utilizando el teléfono cuando es necesario". Cuando las personas en conflicto hablan, es posible percibir dos partes de la historia: la queja hacia el otro, y el pero que indica lo contrario de lo que está pasando o lo que se busca que pase. La doble escucha ayudará al mediador a superar la narrativa del conflicto y así prestar atención a la posible narrativa alternativa. ${ }^{15}$

\section{Parafraseo}

El parafraseo le permite al mediador resumir la narrativa del conflicto que cuentan las partes y validar la comprensión de esta. Además, le facilita generar empatía al ejercitar una escucha activa. Al inicio de la mediación, cuando cada uno de los participantes comparte sus historias, el mediador requiere sintetizar los elementos claves de los hechos del conflicto. Esto se logra parafraseando a las partes en conformidad con lo siguiente:

15. Id. Pág. 7.
1. El momento para hacer el parafraseo es cuando la persona que está hablando termina un "capítulo de su historia" y se dispone a contar otro capítulo. Seguramente, la persona hace una pausa para continuar y ya hay suficientes hechos que resumir.

2. El parafraseo es un reporte de lo dicho y por ello se puede encabezar diciendo: "entiendo que"; "lo dicho por usted hasta el momento es que"; o bien "he escuchado que".

3. La frase del parafraseo debe ser una intervención breve del mediador, la cual idealmente no debería ser superior al $10 \%$ del tiempo empleado por la persona al contar lo que se está resumiendo.

4. El parafraseo debe incluir las palabras clave para la parte autora de la historia, entre ellos verbos y adjetivos. Las palabras y frases deben ser las mismas empleadas por la persona, no una interpretación o "mejor oración" de lo dicho.

5. Con el fin de confirmar que el mediador ha escuchado atentamente y que lo entendido es lo dicho por la persona que ha narrado, al final del parafraseo se debe preguntar a la parte si es correcto o acertado lo mencionado por el mediador. ${ }^{16}$

\section{Replanteo}

El replanteo es una técnica que le permite al mediador facilitar la comunicación apreciativa entre las partes en conflicto. Es común que los involucrados utilicen un lenguaje agresivo para manifestar sus puntos de vista. El conflicto hace que las partes asignen significados negativos a los comportamientos y palabras del otro, de manera que estas se ofenden, reclaman y se comunican perdiendo de vista lo que realmente quieren que suceda (tal vez un cambio en la situación o en el otro). Las palabras pueden ser utilizadas como armas en la batalla que libran las partes y, en ese momento, es cuando el mediador interviene para "atrapar" esos "disparos" y llevarlos al otro con un enfoque que transmita lo que la persona quiere decir, pero desprovisto de ofensas. Si una de las partes dice "él es un mentiroso", "él me robó mi trabajo", el mediador puede intervenir para dirigirse a la persona que lo ha dicho y decirle: "Entiendo que para usted es importante que se hable con la verdad y espera que al presentar un trabajo se den los créditos de quien lo elaboró, ¿fue eso lo que quiso decir?". Como se ve, en el replanteo el mediador matiza un mensaje fuerte hacia una de las partes, el cual puede ocasionar una mayor dificultad en la comunicación. Esto en la medida

\footnotetext{
16. Id. Pág. 269.
} 
en que reconoce lo que la persona quiere decir, pero lo dice de una manera agresiva a causa del conflicto. ${ }^{17}$

\section{Externalización}

La externalización es una técnica que le permite al mediador facilitar en las partes la separación de las personas del conflicto, ya que este se identifica generalmente con la persona contraria. Desde la teoría narrativa, el conflicto es un agente que produce en las partes un cambio en la manera como se percibe una frente a otra. El conflicto se asocia con el ser de la contraparte: el otro es el causante y responsable de las consecuencias negativas del conflicto y no el conflicto en sí, las personas se atacan mutuamente en lugar de unirse y contrarrestar así el conflicto.

En la externalización, el mediador buscará en la narrativa del conflicto identificar un elemento de este (usualmente una emoción o sentimiento), con el cual la persona se relaciona mucho (podría ser la descripción de cómo se siente frente al conflicto). Una vez se detecta ese elemento repetitivo en la narrativa del conflicto, el mediador debe realizar preguntas que lleven a la persona a describir los efectos que ese elemento ha producido en diferentes ámbitos. Así, por ejemplo, si a una persona involucrada el conflicto la ha hecho sentir frustrada, se puede establecer una serie de preguntas: ¿cómo se ha manifestado la frustración? ¿Qué cambios ha producido la frustración? ¿De qué manera la frustración ha afectado su día a día? ¿Qué impacto ha tenido la frustración en su trabajo, casa, relación? Una vez desarrollada la frustración como si fuera un ente aparte de la persona - para esto se utiliza un lenguaje en tercera persona-, es la frustración y no la persona frustrada el objetivo. Luego el mediador debe preguntar por el contrario de la frustración y, una vez la parte lo escoja, se realizan preguntas que desarrollen el sentimiento, emoción o elemento antagónico positivo. Las preguntas buscan que la persona se ubique y se visualice en el escenario donde no está acompañada del conflicto, sino de una historia diferente. La externalización ayuda a que las personas en conflicto se legitimen mutuamente y estén preparadas para trabajar conjuntamente. ${ }^{18}$

\section{Evitar generalizaciones}

Evitar generalizaciones es una técnica que le permite al mediador matizar las generalidades que una

17. Véase Cobb, supra, nota 3.

18. Véase Winslade y Monk, supra, nota 7. Pág. 12. parte hace de la otra a fin de acercarlas. El conflicto, como fenómeno totalizante en las partes, propicia que estas se vean mutuamente como extremos irreconciliables y tiendan a totalizarse la una a la otra. El conflicto figurativamente toma posesión de los actores involucrados y hace ver, interpretar y percibir en el otro un adversario. Cuando una persona afirma: "él siempre es un aprovechado que nunca reconoce el esfuerzo de su equipo de trabajo", el mediador puede intervenir con el fin de hacer una serie de preguntas y pasar del "siempre" y "nunca", a "algunas veces" y "en ocasiones". Las preguntas del mediador podrían ser: ¿hace cuánto que trabajan juntos? ¿Puede contarnos cómo es el proceso de trabajo en equipo en la oficina? ¿Puede darnos algunos ejemplos de circunstancias en las que usted se ha sentido reconocido? ¿En qué ocasiones usted ha tenido la oportunidad de presentar el trabajo que ha elaborado? Con la intervención del mediador, se busca que una de las partes - la cual presenta a la otra como imperfecta- reconozca algunas situaciones en las que la otra no se comporta como suele hacerlo cuando generaliza. ${ }^{19}$

\section{Incluir otras narrativas}

Esta técnica permite al mediador posicionar o ubicar a las partes en el lugar de otros a fin de ampliar sus perspectivas sobre el conflicto y propiciar su reflexión. El conflicto hace que las partes se aferren a sus puntos de vista, razones, argumentos y valores. En la medida en que alguien contradice esas premisas, las partes tienden a fortalecerse y a acrecentar el conflicto. Cuando los involucrados parecen estar anclados en sus narrativas y estar convencidos de que la razón los asiste, el mediador puede hacer preguntas circulares con el fin de llevar a las partes a hablar como otros. Así, el mediador podría intentarlo con las siguientes cuestiones: ¿qué diría su coordinador si estuviera aquí sobre lo que nos acaba de decir? ¿Qué opiniones tendría su colega sobre esta situación? ¿Cómo se comportaría él (contraparte) si usted le hablara de esta manera? ¿Si usted fuera él, qué le gustaría escuchar sobre lo que pasó en la última reunión del comité ejecutivo? Este ejercicio podría ser más directo y pedir la colaboración de las partes para que, por unos minutos, una de ellas intente pensar y hablar como la otra, respondiendo las preguntas del mediador (después se hace lo mismo con la otra parte). Las preguntas circulares ayudan a que los involucrados

19. Véase Cobb, supra, nota 3. 
se muevan de su zona de confort y estén más dispuestos a comprender otras narrativas. ${ }^{20}$

\section{ETAPAS DE LA MEDIACIÓN}

\section{NARRATIVA $^{21}$}

Una vez presentados los supuestos y las técnicas de la mediación narrativa, es necesario articular lo anterior con el método de la mediación. A fin de llevar a cabo una mediación narrativa y ayudar a las partes en conflicto a construir una historia alternativa de respeto, colaboración y reconocimiento mutuo, el mediador las debe invitar a trabajar en sesiones, las cuales pueden desarrollarse por etapas. Es importante tener en cuenta que las etapas de la mediación no son un proceso rígido, el cual tiene que ser cumplido a fin de lograr el objetivo de la mediación. En la práctica, se avanza y retrocede en dichas etapas, pues la división de estos momentos en la mediación tiene más una intención pedagógica cuyo objetivo es presentar los pasos sugeridos para comenzar, avanzar y culminar una mediación narrativa.

La mediación narrativa se compone de siete etapas: inicio; durante la narrativa del conflicto; mapeo de los efectos del conflicto; narrativa alternativa; contraste de la narrativa del conflicto con la narrativa alternativa; preparación del acuerdo; y finalización de la mediación. A continuación se describen cada una de las etapas. ${ }^{22}$

\section{Inicio}

En esta etapa, el mediador buscará generar empatía con las partes y acordar el marco de trabajo en la mediación. La mediación se desarrolla en un espacio físico que el mediador ha preparado y acondicionado para recibir a las partes, el cual debe ser una sala de reunión que garantice la privacidad de las sesiones. El mediador invitará a las personas a un primer encuentro en el que se presentará. Este primer contacto es importante y el mediador buscará que las partes lo legitimen como un tercero válido para llevar a cabo

20. Id.

21. Esta parte procedimental de la mediación narrativa está basada en la obra de Winslade y Monk, supra, nota 7; las sesiones con Cobb, supra, nota 3; y John Winslade. Narrative mediation workshop. (Clase atendida por el autor). Abril 26 de 2012. Arlington, Vancouver, EE.UU.

22. Véase Winslade y Monk, supra, nota 7. la mediación. Al iniciar la mediación, el mediador debe tener en cuenta los siguientes aspectos:

- Presentarse de una manera sencilla indicando que su interés es ayudarles a resolver la situación que los ha traído a la mediación.

- Pedir a cada participante que se presente con su nombre. A fin de empezar a generar equilibrio entre las partes, es recomendable no dirigirse a ellas haciendo referencia a sus cargos o profesiones, ya que esto genera un lenguaje y un trato diferentes. De la misma manera, el mediador esperaría que lo llamaran por su nombre también.

- El mediador felicita a las personas por haber escogido la mediación como una forma de resolver la situación que les preocupa o el conflicto, así como el haber aceptado la invitación a esta primera sesión.

- El mediador presentará qué es la mediación, la manera de desarrollarse, el papel que cumple el mediador, los beneficios ylos efectos delos acuerdos. En esta parte es fundamental que los participantes validen la información. Para esto, el mediador les puede pedir que en sus propias palabras mencionen cada uno de los puntos anteriores, de manera que se corrobore que todos tienen claridad sobre el contexto y los alcances de la mediación.

- Un punto importante en el inicio es que el mediador les informe a las personas involucradas que su labor como mediador dependerá de la voluntad de ellas de permitir servirles; así como en cualquier momento de la mediación si ellas perciben que su trabajo es parcializado hacia alguna de las partes, se lo hagan saber, de manera que puedan dialogar y tomar las decisiones a que haya lugar. Son las partes quienes deben estar empoderadas de la mediación y sus resultados.

- A fin de desarrollar la mediación, es importante construir unos acuerdos iniciales con las partes acerca de las reglas que se deberían cumplir. Para continuar con el empoderamiento de las partes, las reglas deben ser resultado de sus propuestas, del acuerdo y el compromiso de cumplirlas. Este punto le permitirá al mediador resaltar cómo a pesar de la situación por la cual ellas están pasando, es posible dialogar y lograr acuerdos.

- Con el propósito de entrar en materia, el mediador debe invitar a las partes a que cualquiera de ellas inicie y cuente lo que ha sucedido. Para la mediación narrativa, la persona que empieza contando su historia (narrativa del conflicto) tiene ventajas, ya que la segunda persona en intervenir seguramente buscará justificar, defenderse o contrarrestar lo ya dicho y no compartir su historia independientemente. Así, para el mediador narrativo, un paso 
estratégico es invitar a que hable primero quien desee y al segundo apoyarlo para que comparta su punto de vista libremente. ${ }^{23}$

\section{Durante la narrativa del conflicto}

En la segunda etapa de la mediación narrativa, el mediador facilitará en cada una de las fases que los involucrados cuenten su narrativa del conflicto con todos los detalles posibles. En esta etapa es más evidente el uso de algunas técnicas como:

- Doble escucha. Desde el inicio de la mediación, el mediador debe tener en cuenta el contraste entre la narrativa del conflicto y una posible narrativa alternativa.

- Parafraseo. Durante los capítulos de la narrativa del conflicto el mediador debe hacer un parafraseo, de modo que al final obtenga una síntesis de las diferentes versiones de la historia.

- Incluir otras narrativas. Una vez las personas han contado sus narrativas del conflicto, el mediador puede hacerle a cada una de ellas preguntas que les permitan situarse en los zapatos de otras personas.

- Externalización. Después de tener la narrativa del conflicto, el mediador puede, en una sesión individual o conjunta, trabajar la externalización con el fin de separar la persona del problema.

- Replanteo. En caso de necesitarlo, el mediador puede hacer replanteos de las ofensas o mensajes fuertes de las partes.

- Evitar generalizaciones. En caso de necesitarlo, el mediador puede intervenir para matizar las generalizaciones de las partes.

En la narrativa del conflicto, es clave que el mediador facilite la narración al hacer preguntas abiertas y preguntas de aclaración. En esta etapa no se debe buscar la confirmación de las hipótesis del mediador, es más bien la oportunidad para una escucha activa de la historia cuyos autores son las partes. Tampoco en esta etapa se deben explorar soluciones, propuestas ni pasos a seguir. El mediador debe estar concentrado en contar con toda la información disponible acerca de lo que ha sucedido. No es una búsqueda de la verdad, la razón o las pruebas: se trata es de obtener la perspectiva completa de las partes.

23. Véase Sara Cobb. Narrative "Braiding" and the Role of Public Officials in Transforming the Public's Conflicts. NARRATIVE AND Conflict: Explorations in Theory and Practice 1. Diciembre de 2013. Págs. 4-30

\section{Mapeo de los efectos del conflicto}

Una vez se ha completado la etapa de contar la narrativa del conflicto, el mediador debe tomarse un tiempo para mapear los efectos que el conflicto ha tenido en las partes. A fin de lograr esto, se deben seguir las siguientes pautas:

- El mediador debe preguntar a cada participante por un nombre para el conflicto o la situación general.

- Apoyado con un mapa mental, el mediador debe recoger los efectos, consecuencias e impactos del conflicto en las partes (el nombre que le han dado al conflicto debe ir en el centro). Algunos de estos efectos seguramente han sido comentados por las partes, de manera que el mediador puede preguntar por ellos concretamente. La externalización aplicada por el mediador puede ayudar con información para complementar el mapa mental.

- Los efectos, consecuencias e impactos se pueden desarrollar en diferentes escenarios (laboral, familiar, comunitario, personal). También se puede trabajar en diferentes tiempos (pasado, presente y uno futuro condicional que contemple lo que seguiría pasando si no se cambia la dinámica en que el conflicto los tiene envueltos).

- Una vez terminado el mapa mental, el cual puede recogerse en una cartelera o diapositiva, el mediador lo presentará a las partes como un resumen de todo lo dicho hasta el momento.

- Es importante que el mediador utilice un lenguaje externalizado (tercera persona del singular) para referirse al conflicto, utilizando el nombre dado por las partes. El mediador no se enfoca en lo que las partes han hecho, sino en lo que el conflicto les ha hecho.

\section{Narrativa alternativa}

Hasta el momento se han expuesto las narrativas del conflicto y los efectos del conflicto. Con esta información, el mediador debe ayudar a las partes a construir una narrativa alternativa. Para esto, se deben tener en cuenta los siguientes aspectos:

- En conformidad con los resultados de la aplicación permanente de la técnica de la doble escucha, el mediador debe tener un borrador de narrativa alternativa. Esta versión le servirá como guía de facilitación de esta etapa.

- Con el borrador de la doble escucha, el mediador puede complementarla con las partes de la narrativa del conflicto que contradicen el conflicto en sí; en otras palabras, las cosas que funcionaron antes entre 
las partes y que les permitirían hacer el cambio de nuevo. También se pueden tener en cuenta los diferentes elementos de la historia conflictiva en los que las partes se legitiman mutuamente, identificar los valores que subyacen entre las partes, los intereses y necesidades comunes, y tener presente los momentos en que las partes rechazaron el conflicto, así como considerar los significados que las partes dan a las situaciones que las llevaron al conflicto. Todos estos elementos son diferentes insumos que sirven para estructurar una narrativa alternativa desde la perspectiva del mediador.

- El mediador debe invitar a las partes a pensar en un nombre para una historia diferente a su situación o conflicto. Una vez dado este nombre, el mediador facilita el desarrollo de dicha historia o narrativa alternativa.

- Para desarrollar la narrativa alternativa, es importante que el mediador maneje diferentes tipos de preguntas. Las preguntas que lo ayudarían son las siguientes:

-Preguntas con resultados únicos. Empoderan a las personas y las ayudan a verse fuera del conflicto; por ejemplo: ¿qué te ha motivado para que en diferentes oportunidades hayas intentado hablar con tu jefe sobre esta situación?

-Preguntas que cuentan una sola vez. Profundizan en las situaciones excepcionales del conflicto; por ejemplo: ¿cómo te sientes cuando logras ponerte de acuerdo con tu compañero de trabajo y cumplir con las metas trazadas?

-Preguntas de redescripción única. Invitan a pensar en el comportamiento y cómo quisieran que fuera la relación; por ejemplo: si ustedes dos continuaran el diálogo como lo han venido haciendo en estas dos últimas reuniones, ¿cómo sería el trabajo de ustedes en la empresa?

-Preguntas de posibilidad única. Se enfocan en el futuro positivo; por ejemplo: si se cumpliera lo que ustedes han acordado hoy, ¿cómo sería el ambiente laboral en seis meses?

-Preguntas circulares únicas. Buscan ubicar las partes en el contexto social en el que ellas viven; por ejemplo: si estuviera presente el director de la organización, ¿qué opinión nos daría sobre la situación en su grupo y la manera como ustedes quisieran manejarla?

- El mediador invitará a las partes a desarrollar la narrativa alternativa usando el nombre que le han dado y a través de un mapa mental con los diferentes aspectos positivos que apliquen (laboral, familiar, comunitario, personal, presente y futuro). Para esto, el mediador se apoyará en los tipos de preguntas presentados.

- La narrativa alternativa debe apuntar principalmente lo que las personas buscan y les permita lograr respeto, colaboración y legitimación mutua. De igual forma, la narrativa alternativa del mediador le servirá como guía para motivar a las partes a construir conjuntamente su nueva narrativa.

\section{Contraste de la narrativa del conflicto con la narrativa alternativa}

Las partes han trabajado con la ayuda del mediador dos cosas: la narrativa del conflicto y la narrativa alternativa. El mediador en esta etapa hará una presentación de una y otra. Es útil contar con herramientas visuales que les permitan a los involucrados en el conflicto observar las dos en paralelo. Una vez el mediador ha hecho lo anterior, procederá a preguntarles: ¿cuál de las dos situaciones prefieren? Aunque esta pregunta induzca una respuesta obvia, es muy importante que las partes respondan abiertamente con cuál se quedan. Es un momento crucial para continuar con la siguiente fase, pues las partes deben comprometerse - si así lo quieren - con la narrativa alternativa. Una vez ellas han elegido la narrativa alternativa, el mediador las invita a hablar cómo serían las cosas en el futuro si esa narrativa fuera una realidad.

\section{Preparación del acuerdo}

La narrativa alternativa es el camino que las partes han trazado para el futuro y será la estructura del acuerdo que ellos construyan mutuamente. Hasta el momento, las partes han pasado por varias fases que les han permitido estar preparadas para hablar de propuestas, soluciones y futuro. Si no es posible avanzar en esta fase, es porque seguramente las partes deben trabajar más sobre su narrativa del conflicto o narrativa alternativa.

Si la narrativa alternativa es el camino, el mediador ayudará a las partes en esta etapa a llenar de contenido esa ruta trazada, esto es, establecer los compromisos concretos que harán posible hacer realidad su narrativa alternativa. No es suficiente visualizar una meta si no se definen acciones cumplibles para avanzar. Asimismo, el mediador facilitará a las partes el diálogo a fin de que pongan el asfalto, la señalización y la iluminación, por así decirlo, de su acuerdo. Es importante tener en cuenta que el 
acuerdo debe procurar que las personas se respeten, colaboren y legitimen mutuamente, lo cual es el objetivo de la mediación narrativa.

\section{Finalización de la mediación}

En la mediación narrativa se busca que los acuerdos entre las partes sean verbales y se valore la palabra como compromiso. El mediador cierra la mediación felicitando a las partes por el camino recorrido, ya que son ellas las protagonistas del conflicto y su solución, así como las autoras de sus narrativas $y$, por tanto, deben sentirse empoderadas de su narrativa alternativa. Antes de terminar la sesión el mediador les informará cómo, en aras de acompañarlas más adelante en la realización de sus compromisos, él entrará en contacto con ellas a fin de realizar un diálogo y evaluar los avances.

El trabajo en la mediación narrativa no termina con el acuerdo: el significado del acuerdo - desde una perspectiva narrativa - es el comienzo de un cambio que las partes han decidido hacer, con el objetivo de que la historia que han venido nutriendo de conflicto se transforme por medio de una narrativa alternativa. El acuerdo no termina un conflicto, no lo desaparece, no hace que se pase del conflicto a la paz; el acuerdo es un compromiso hacia el futuro en el que las dos partes deben respetarse, colaborar y legitimarse para que posteriormente ellas puedan evidenciar que es posible un cambio, ya que así ellas mismas lo quisieron.

Este es el comienzo de una nueva historia, en la cual se espera que las partes cuenten con respeto, colaboración y reconocimiento mutuo.

\section{CONCLUSIONES}

La mediación narrativa es un método de resolución de conflictos que permite a las partes en una organización gestionar, con la ayuda de un mediador, la superación de su conflicto. Las controversias afectan gravemente el desarrollo de la misión de una organización y, por tanto, es necesario que todas las partes que integran una entidad pública o privada cooperen mutuamente a fin de encontrar soluciones a los problemas que se presentan como un fenómeno natural en las relaciones humanas. La mediación es el escenario ideal para que las partes dialoguen y logren acuerdos que modifiquen sus narrativas del conflicto por narrativas alternativas en su entorno laboral. Así, la mediación se convierte en la principal herramienta para promover la convivencia y con ello mejorar el clima laboral.

Los conflictos en las empresas y entidades públicas son historias o narrativas que han crecido entre las partes, las afecta y polariza, pues les hace percibir que aquello que busca cada una es incompatible con lo que busca la otra. Al implementar programas de mediación organizacional, las instituciones estarán en capacidad de romper la espiral del conflicto y de esta forma empezar una negociación constante de las narrativas de las partes, de manera que se transformen por unas de respeto, colaboración y reconocimiento mutuo, aspectos fundamentales para el trabajo en equipo. Con el fin de conseguir este propósito, la alta gerencia debe ser consciente del daño y el costo de los conflictos en el interior de la organización y debe promover la mediación como herramienta de diálogo.

Para llevar a cabo una mediación narrativa, el mediador debe conocer la teoría, dominar la práctica y reflexionar sobre su actuar. La teoría de este tipo de mediación se basa en lo que las teorías narrativas informan sobre el conflicto, y la forma de abordarlo y superarlo. La práctica hace referencia a las técnicas de intervención en los conflictos y a las etapas que se deben seguir y agotar para llevar a cabo una mediación. La reflexión es el constante interés del mediador por preguntarse y cuestionarse sobre qué propone la teoría y su aplicación práctica, a fin de generar lecciones aprendidas y mejorar la mediación. Con lo anterior, los operadores de la mediación narrativa en las organizaciones podrán brindar una mediación ética y profesional a sus colegas de trabajo.

Si bien el conocimiento y desarrollo de la mediación narrativa en Colombia y Latinoamérica es incipiente, en países como Estados Unidos la mediación narrativa ha demostrado sus potencialidades como método de resolución de conflictos en contextos institucionales públicos y privados. Para el debido desarrollo de la mediación con enfoque narrativo se requiere, por un lado, el aporte de la academia en la formación (las técnicas y el método narrativo de mediadores), que promueva la mediación dentro de las organizaciones. Por otro lado, se requiere también el apoyo decidido de los líderes en la toma de decisiones, de manera que el diálogo sea el instrumento primordial en la resolución de conflictos dentro de las empresas. La mediación narrativa es la oportunidad que tienen las personas de construir historias de respeto, colaboración y reconocimiento mutuo; sólo así se transforma la cultura del litigio y la violencia que muchas veces impera en nuestras sociedades. 


\section{REFERENCIAS}

Gregory Alonso Pirio. Journalism and HIV. Lessons from the Frontline of Behavior Change Communication in Mozambique. Eds. Rafael Obregón y Silvio Waisbord. THE HANDBOOK OF GLOBAL HEALTH COMMUNICATION. Wiley-Blackwell. (2012). Págs. 426-443.

John Winslade. NarRative MEdiation: A NEW APPROACH TO CONFLiCt RESOlution. Jossey-Bass. (2000).

John Winslade y Gerald Monk. Practicing narrative MEDIATION: LOOSENING THE GRIP OF CONFLICT Jossey-Bass. (2008).

John Winslade y Michael Williams. SAfe AND PEACEful SCHOOLS: ADDRESSING CONFLICT AND ELIMINATING VIOLENCE. Corwin. (2012).

John Winslade. Narrative mediation workshop. (Clase atendida por el autor). Abril 26 de 2012. Arlington, Vancouver, EE.UU.

John Winslade. Welcome letter. NARRATIVE AND Conflict: Explorations in Theory and Practice 1. Diciembre de 2013. Págs. 1-3.

Hilde Lindemann. Damaged identities, Narrative REPAIR. Cornell University Press. (2001).

María Pía Lara. Narrating evil: a postmetaphysical THEORY OF REFLECTIVE JUdGMENT. Columbia University Press. (2007).
Mohammed D. Cherkaoui. Civilians, pundits, and the mediatized ideology. CIVILIANS AND MODERN WAR : ARMED CONFLICT AND THE IDEOLOGY OF VIOLENCE. Routledge. (2012).

Robert A. Baruch y Joseph P. Folger. LA PROMESA DE LA MEDIACIÓN: CÓMO AFRONTAR EL CONFLICTO MEDIANTE LA REVALORIZACIÓN Y EL RECONOCIMIENTO. Granica. (1996).

Roger Fisher, William Ury y Bruce Patton. iSí, DE ACUERDO!: CóMO NEGOCIAR SIN CEDER. Norma. (1993).

Sara Cobb. Narrative mediation working group. (Clase atendida por el autor). Abril 15 de 2011. Arlington, Vancouver, EE.UU.

Sara Cobb. SPEAKING OF Violence: THE POlitics AND POETICS OF NARRATIVE DYNAMICS IN CONFLICT RESOLUTION. Oxford University Press. (2013).

Sara Cobb. Narrative "braiding" and the role of public officials in transforming the public's conflicts. NARRATIVE AND Conflict: Explorations in Theory and Practice 1. Diciembre de 2013. Págs. 4-30.

Solon Simmons. The eClipse of Equality: ARguing AMERICA ON MEET THE PRESS. Stanford University Press. (2013) 